July 2016, Volume 1, Issue 2 (20-22)

\title{
Venous Obstruction Following Pacemaker or Implantable Cardioverter-Defibrillator Implantation, Mini Review
}

\author{
Mohammad Ali Akbarzadeh 1,2,* \\ ${ }^{1}$ Cardiovascular Research Center, Shahid Beheshti University of Medical Sciences, \\ Tehran, Iran \\ ${ }^{2}$ Department of Cardiology, School of Medicine, Beheshti University of Medical \\ Sciences, Tehran, Iran \\ * Corresponding author: Mohammad Ali Akbarzadeh, Cardiovascular Research \\ Center, Shahid Modarres Hospital, Saadat Abad Street, Tehran, Iran. Tel: +98- \\ 9173171001,E-mail:akbarzadehali@yahoo.com
}

DOI: $10.20286 /$ ijcp-010201

Submited: 04.09.2015

Accepted: 05.20.2015

\section{Keywords:}

Upper Extremity Deep Vein Thrombosis

Pacemaker, Artificial

Defibrillators

(C) 2016. International Journal of Cardiovascular Practice.

\begin{abstract}
Venous obstruction is relatively frequent following permanent pacemaker or implantable cardioverter-defibrillator (ICD) implantation. However, most of them are asymptomatic. Although the exact risk factor for this complication is not known, number of leads, heart failure and infection may prone the patient to this complication. The goal standard for detection of vein stenosis is venography; however, ultrasound sonography has an acceptable accuracy. Anticoagulant therapy may be considered for symptomatic patients. For device upgrading, non-functional leads removal, venoplasty and rarely surgical treatment may be indicated.
\end{abstract}

\section{INTRODUCTION}

With the growing numbers of pacemaker and implantable cardioverter defibrillator (ICD) implantations, obstruction of the access vein is detected more in these patients. Development of thrombosis and obstruction is a multifactorial process. Ligation of the access vein during cut-down approach, slowing of the flow in the vein due to decreased luminal area by the leads, and endothelial trauma caused by pacemaker leads, cause prothrombotic and inflammatory responses which may cause thrombus formation and subsequent scarring that lead to obstruction [1].

In view of the diagnosis of lead-related thrombosis, two types of obstructions can be distinguished; few patients develop clinically manifest obstructions with overt symptoms and signs, such as pain, tenderness, edema, warmth, paresthesia or bluish discoloration of ipsilateral arm. Pulmonary embolism and superior vena cava (SVC) syndrome are the two major but rare complications of lead-related venous thrombosis in this group [2]. However, most of the patients have subclinical obstructions and in the absence of signs or symptoms, they are detected at follow-up procedures such as system revision and lead extraction or during screening diagnostic imagines (Fig 1) [2].

It shows implantable cardioverter defibrillator lead fracture and totally obstruction of the left subclavian vein with collateral formation in a 48 years old lady, 4 years after device implantation.

In prospective and retrospective studies, the incidence of significant venous obstruction was reported as $13.7-45 \%$ following endocardial lead insertion. However, clinically manifested obstruction is relatively rare (1-3\%) [3-5].

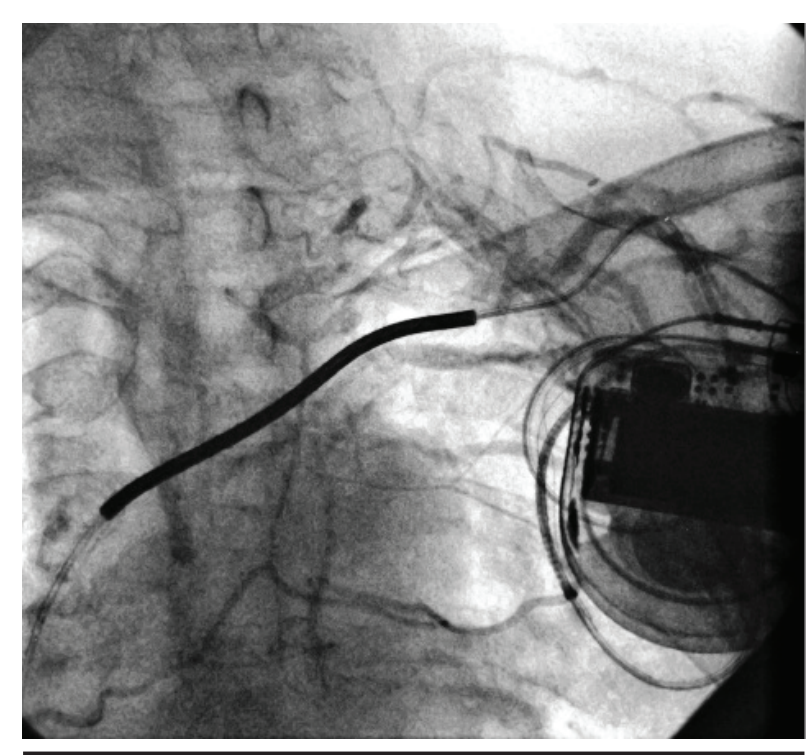

Figure 1: Fluoroscopy and venography in AP View

\section{RISK FACTORS}

Data about risk factors of venous obstruction after pacemaker implantation are conflicting and show many discrepancies. These conflicts may be due to different imaging modalities used for the detection of obstruction, length of their follow-ups and different portions of each type of cardiac rhythm management device (PPM, ICD or CRT). In almost all the investigations, age, gender, smoking, lead size and cephalic 
vs. subclavian approach were not found as risk factors for venous obstruction. Some studies determined number of leads, heart failure, female hormone use, personal history of venous thrombosis, and infection as risk factors. However, some other studies did not approve these correlations [3, 6, 7]. Prophylaxis warfarin and antiplatelet consumption may lower the probability of venous stenosis [8].

\section{DIAGNOSIS}

\section{Venography}

Contrast venography is recognized as the standard method for diagnosis and is mostly performed before upgrading procedures. This method requires the use of ionizing radiation [9]. It can be performed by the injection of contrast medium through a large peripheral intravenous line [3].

\section{Ultrasound Sonography}

Ultrasound is most often used in patients with clinical signs or symptoms, because it is non-invasive, can easily be performed at the bedside, and the patient does not get exposed to contrast medium or radiation. Non-compressibility, direct visualization of thrombus in the venous lumen and evaluation of blood-flow can be used to establish the presence or absence of thrombosis in ultrasound sonography [10].

\section{Computed Topography}

Spiral computed topography (CT) venography is also an accurate diagnostic tool which can detect deep vein thrombosis and require lesser amount of contrast medium compared to conventional venography. However, CT scan has not been validated for central chest veins [1]. Nonetheless, there are some reports aiming that this imaging modality can be helpful [11-13].

\section{Magnetic Resonance Angiography}

Magnetic resonance angiography was only evaluated in few studies with small sample sizes [10]. It is a non-invasive imaging modality that has acceptable accuracy for the detection of thrombus in the central thoracic veins and does not require contrast medium. Unfortunately, it is relatively contraindicated in the presence of a pacemaker or ICD [14].

\section{MANAGEMENT}

The strategy for the management of these patients depends on their signs, symptoms and clinical statuses. Since most patients with occlusion of subclavian vein are asymptomatic, and also few of them will require lead revision, or an ipsilateral lead insertion in future, obstruction does not develop any problem and does not need any management. A short-term warfarin therapy for 3-6 months is used after acute episodes of upper extremity deep vein thrombosis; however, there is no current recommendation to use warfarin as a primary prevention after pacemaker leads implantation [1]. In the case of upgrade procedure, non-functional, old lead removal can restore the patency of previously occluded veins [15]. Successful venoplasty of SVC, innominate or subclavian veins that may allow subsequent lead insertion was also reported [16]. In a few case reports, surgical treatments of pacemaker-related SVC syndromes that did not respond to treatment with anticoagulation or had contraindications to endovascular procedure were performed successfully [17]. Surgical management requires thoracotomy and has high morbidity; so, should be considered as the last choice.

\section{CONFLICTS OF INTEREST}

There was no conflict of interests for the present study.

\section{REFERENCES}

1. Rozmus G, Daubert JP, Huang DT, Rosero S, Hall B, Francis C. Venous thrombosis and stenosis after implantation of pacemakers and defibrillators. J Interv Card Electrophysiol. 2005;13(1):9-19. DOI: 10.1007/ s10840-005-1140-1 PMID: 15976973

2. Engelberger RP, Kucher N. Management of deep vein thrombosis of the upper extremity. Circulation. 2012;126(6):768-73. DOI: 10.1161/ CIRCULATIONAHA.111.051276 PMID: 22869858

3. Haghjoo M, Nikoo MH, Fazelifar AF, Alizadeh A, Emkanjoo Z, Sadr-Ameli MA. Predictors of venous obstruction following pacemaker or implantable cardioverter-defibrillator implantation: a contrast venographic study on 100 patients admitted for generator change, lead revision, or device upgrade. Europace. 2007;9(5):328-32. DOI: 10.1093/ europace/eum019 PMID: 17369270

4. Antonelli D, Turgeman Y, Kaveh Z, Artoul S, Rosenfeld T. Short-term thrombosis after transvenous permanent pacemaker insertion. Pacing Clin Electrophysiol. 1989;12(2):280-2. PMID: 2468137

5. Da Costa SS, Scalabrini Neto A, Costa R, Caldas JG, Martinelli Filho $\mathrm{M}$. Incidence and risk factors of upper extremity deep vein lesions after permanent transvenous pacemaker implant: a 6-month follow-up prospective study. Pacing Clin Electrophysiol. 2002;25(9):1301-6. PMID: $\underline{12380764}$

6. van Rooden CJ, Molhoek SG, Rosendaal FR, Schalij MJ, Meinders AE, Huisman MV. Incidence and risk factors of early venous thrombosis associated with permanent pacemaker leads. J Cardiovasc Electrophysiol. 2004;15(11):1258-62. DOI: 10.1046/j.1540-8167.2004.04081.x PMID: 15574174

7. Pauletti M, Di Ricco G, Solfanelli S, Marini C, Contini C, Giuntini C. Venous obstruction in permanent pacemaker patients: an isotopic study. Pacing Clin Electrophysiol. 1981;4(1):36-42. PMID: 6171790

8. Costa R, Da Silva KR, Rached R, Martinelli Filho M, Carnevale FC, Moreira LF, et al. Prevention of venous thrombosis by warfarin after permanent transvenous leads implantation in high-risk patients. Pacing Clin Electrophysiol. 2009;32 Suppl 1:S247-51. DOI: 10.1111/j.15408159.2008.02295.x PMID: 19250106

9. Saseedharan $S$, Bhargava $S$. Upper extremity deep vein thrombosis. Int J Crit Illn Inj Sci. 2012;2(1):21-6. DOI: 10.4103/2229-5151.94891 PMID: 22624098

10. Di Nisio M, Van Sluis GL, Bossuyt PM, Buller HR, Porreca E, Rutjes AW. Accuracy of diagnostic tests for clinically suspected upper extremity deep vein thrombosis: a systematic review. J Thromb Haemost. 2010;8(4):684-92. DOI: 10.1111/j.1538-7836.2010.03771.x PMID: 20141579

11. Lonyai A, Dubin AM, Feinstein JA, Taylor CA, Shadden SC. New insights into pacemaker lead-induced venous occlusion: simulation-based investigation of alterations in venous biomechanics. Cardiovasc Eng. 2010;10(2):84-90. DOI: 10.1007/s10558-010-9096-x PMID: 20514553

12. Tello R, Scholz E, Finn JP, Costello P. Subclavian vein thrombosis detected with spiral CT and three-dimensional reconstruction. AJR Am J Roentgenol. 1993;160(1):33-4. DOI: 10.2214/ajr.160.1.8416641 PMID: 8416641

13. Senthilvel E, Papadakis A, Jain V, Bruner J. Pacemaker induced superior vena cava syndrome: a case report. Cases J. 2009;2:6463. DOI: 10.4076/1757-1626-2-6463 PMID: 19829810

14. Junttila MJ, Fishman JE, Lopera GA, Pattany PM, Velazquez DL, Williams AR, et al. Safety of serial MRI in patients with implantable cardioverter defibrillators. Heart. 2011;97(22):1852-6. DOI: 10.1136/ heartinl-2011-300153 PMID: 21873440

15. Smith MC, Love CJ. Extraction of transvenous pacing and ICD leads. 
Pacing Clin Electrophysiol. 2008;31(6):736-52. DOI: 10.1111/j.15408159.2008.01079.x PMID: 18507548

16. Teo N, Sabharwal T, Rowland E, Curry P, Adam A. Treatment of superior vena cava obstruction secondary to pacemaker wires with balloon venoplasty and insertion of metallic stents. Eur Heart J.
2002;23(18):1465-70. PMID: 12208227

17. Barakat K, Robinson NM, Spurrell RA. Transvenous pacing lead-induced thrombosis: a series of cases with a review of the literature. Cardiology. 2000;93(3):142-8. DOI: 10.1159/000007018 PMID: $\underline{10965084}$ 